\title{
Hybrid Books for Interactive Digital Storytelling: Connecting Story Entities and Emotions to Smart Environments
}

\author{
No Author Given \\ No Institute Given
}

\begin{abstract}
Nowadays, many people use e-books, having high expectations with respect to their reading experience. In the case of digital storytelling, enhanced e-books can connect story entities and emotions to real-world elements. In this paper, we present the novel concept of a Hybrid Book, a generic Interactive Digital Narrative (IDN) artifact that requires seamless collaboration between content and smart devices. To that end, we extract data from a story and broadcast these data in RDF as Linked Data. Smart device services can then receive and process these data in order to execute corresponding actions. By following open standards, a Hybrid Book can also be seen as an interoperable and sustainable IDN artifact. In addition, according to our user-based evaluation, a Hybrid Book makes it possible to provide human sensible feedback while flipping pages, enabling a more enjoyable reading experience. Finally, the participants also showed a positive willingness to pay, thus making it possible to generate more revenue for authors and publishers.
\end{abstract}

Keywords: e-Books, EPUB 3, Interactive Digital Narrative, Semantic Web, Smart Living Environments

\section{Introduction}

Stories can be presented in several ways, for instance through the use of movies and e-books (that is, digital books). The definition of an e-book, as discussed by [31], contains both a static and a dynamic component: whereas the static component states that "an e-book is a digital object with textual and/or other types of content", the dynamic component expresses that "an e-book can have technology-dependent features that make it more interactive and dynamic than its paper counterpart". The rise of e-books brings opportunities for new reading experiences. One such reading experience may consist of having interaction with the physical environment when making use of a so-called "Hybrid Book".

Anastasiades [2] defines the term "Hybrid Book" as "the best combination of use of traditional printed books and multiple potentials offered by a virtual educational environment". We propose to generalize the term "Hybrid Book" to a paper or digital book that extends into the "physical or digital realm". Specifically, according to our definition, a Hybrid Book can take the form of an 
e-book that facilitates interaction with the physical and/or digital world, or it can take the form of a paper book that has been enriched using digital content (e.g., through the use of augmented reality).

In the above context, it is worth pointing out that EPUB 3 [15] is a powerful format for both representing and presenting e-books [10]. This format is based on HTML5, JavaScript, and CSS. Furthermore, this format supports the desired attributes of enhanced publications [35], for instance making it possible to integrate multimedia features and semantic annotations using RDFa [13] or microdata [14]. Moreover, EPUB 3 makes it possible to connect book content to networked services [11].

Nowadays, people can equip their living environments with smart devices, including smart lights and smart thermostats. Furthermore, people can control these smart devices either locally or remotely. We believe that reading stories is an activity that can be made more attractive in such smart living environments.

The contributions of this paper are as follows. We document an approach to connect story entities and emotions to (1) relevant digital content that is open in nature and (2) to smart devices, taking advantage of Digital Publishing tools (e.g., EPUB 3) and Semantic Web technology (e.g., SPARQL and ontology reasoning). In this context, we see an author as the person who acts as the designer of a story and as the person who decides what the desired interactions are. Furthermore, we investigate the way story entities (e.g., people, locations, and animals) and emotions can be represented within an e-book and how these entities and emotions can facilitate interaction with a smart living environment (e.g., change of lights and sounds). To that end, we explore the way a book can broadcast required data to smart devices and how these devices can interpret the data and perform the right actions. Furthermore, we present a prototype Hybrid Book that allows its readers to have a more enjoyable reading experience. Additionally, we evaluate this prototype Hybrid Book, taking into account key aspects of Interactive Digital Narratives (IDNs) [18].

The remainder of this paper is organized as follows. In Section 2, we review related work. In Section 3, we discuss characteristics of both IDN applications and EPUB 3-based Hybrid Books. In Section 4, we explain our approach for connecting story entities and emotions to relevant digital content and smart devices. We subsequently evaluate a prototype Hybrid Book in Section 5. Finally, we present our conclusions and a number of directions for future research in Section 6 .

\section{Related Work}

In this section, we review a number of research efforts and best practices related to Interactive Digital Narratives. In particular, we first pay attention to interactive story books in smart environments. Next, we focus on semantic content representation and reasoning about entities and emotions in interactive stories. 


\subsection{Interactive Digital Narratives in Smart Environments}

Koenitz [19] defined an Interactive Digital Narrative (IDN) as follows: an "expressive narrative form in digital media implemented as a computational system containing potential narratives and experienced through a participatory process that results in products representing instantiated narratives". Key characteristics of a digital narrative are as follows [20]: "method of introduction (explorative vs. consecutive)", "narrative world (parallel vs. interwoven)", "narrative path (fixed-path vs. conditional-path)", "narrative presentation (text-based vs. graphic-based)", and "point of view (uni-character vs. multi-characters)".

As an example of already existing applications, we refer to two iPad applications: Philips Disney StoryLight ${ }^{1}$ and Light Stories ${ }^{2}$. These applications take on the role of an e-book, enabling the reader to change the color of the Philips hue light ${ }^{3}$ by touching screen elements. Besides the usage of lights for creating interactive stories, sound can be used as a complementary channel as well, given that the use of sound brings fantasy [23].

Furthermore, Nakevska et al. [24] proposed the concept of interactive storytelling in a mixed reality in which users do not directly use a computer and interaction devices. They provided three different stages, equipping all stages with sensory devices originating from a so-called ALICE installation [4], thus enriching these stages with different relevant sounds. The participants had to physically follow the aforementioned stages, having an exploratory experience during storytelling. In addition, the authors investigated immersiveness and user experience, finding that sound affects self-location in a storytelling environment.

The above discussed approaches, however, are proprietary in nature, while the Hybrid Book solution we propose is a generic approach that can be integrated in e-books using the standardized EPUB 3 format $^{4}$.

\subsection{Semantic Representation and Reasoning}

Semantic Web technologies aim to collect, structure, and recover linked data. In what follows, we describe a number of WC3 Semantic Web standards ${ }^{5}$ that can be used to enrich story books, hereby creating interactive storytelling experiences.

Resource Description Framework (RDF) [17] is a general-purpose framework for representing Web information, expressing data in a machine-understandable format. RDF in attributes (RDFa) [13] adds a set of attribute-level extensions to (X)HTML documents, with the goal of including RDF data inside these documents. Terse RDF Triple Language (Turtle) [5] is a serialization format for RDF. An RDF query language such as SPARQL [27] is able to retrieve and manipulate data stored in RDF format. An ontology is "a specification of a representational

\footnotetext{
1 http://disneystories.com/app/disney-storytime/

${ }^{2}$ https://itunes.apple.com/us/app/light-stories/id692093472?mt=8

${ }^{3}$ http://www2.meethue.com/en-us/

${ }^{4}$ For the latest status on EPUB 3 support, please see http://epubtest.org/.

${ }^{5}$ http://www.w3.org/standards/
} 
vocabulary for a shared domain of discourse - definitions of classes, relations, functions, and other objects" [28]. The Web Ontology Language (OWL) [34] is a semantic markup language for publishing and sharing ontologies on the World Wide Web.

Enriching HTML pages of e-books by using RDFa and microdata makes story content machine-readable, thus facilitating the execution of queries against this content. Also, sets of vocabularies can be specified in ontologies. These sets of vocabularies can be used for extraction and reasoning purposes, with the aim of deriving new data. These new data can then be used for different goals, such as activating smart devices or finding relevant songs in an online sound repository. The Linked Data Fragments client and server [36] are two libraries for affordable and reliable querying of linked data with SPARQL.

Different stories often have similar themes, items, and characters. The Stories ontology ${ }^{6}$ was developed in collaboration with the BBC, with the aim of linking items together in order to make stories navigable and discoverable. Onyx [30] is a linguistic Linked Data approach for representing emotions. In particular, Onyx is a semantic emotion vocabulary that focuses on lexical resources and emotion analysis services. The Onyx model includes EmotionML [3] vocabularies. EmotionML is a general-purpose emotion annotation and representation language using XML. As we use HTML files for representing story content, EmotionML is not suitable for supporting different emotions in EPUB 3-based e-books.

Considering the aforementioned related work, our proposed solution consists of a generic way to represent the content of interactive story books. In particular, we make use of RDF for broadcasting story entities and emotions. Furthermore, we created an ontology that allows describing the relationship between story entities and emotions on the one hand, and actions for activating smart devices on the other hand. In addition, our ontology allows mapping emotions to corresponding colors [25].

\section{Hybrid Books as IDN Artifacts}

In this section, we discuss the possibility of EPUB 3-based Hybrid Books to act as IDN artifacts. Considering the definition and characteristics of an IDN [19], we focus on a "fixed-path" narrative (as is commonly done for fiction books).

In the traditional way of creating fiction books, an author writes a story in the form of a text and a publisher distributes the text as it is. Such fiction books are passive [21] in nature. However, these passive books can be converted to interactive e-books (e.g., Hybrid Books) by integrating interactivity and multimedia features. In advanced cases, the story entities and the emotions conveyed by these e-books can be connected to both the digital and the physical world.

IDN artifacts can be classified based on the presence of interactive features. For example, they can be mapped along the axes of exploration (story versus system) and control (user control versus system control). In addition, they can

\footnotetext{
${ }^{6}$ http://www. contextus.net/stories
} 
be mapped by taking into account criteria like "agency (the variety of actions that a user can undertake)", "narrative complexity (the quantity of concurrent narrative developments)", and "dramatic agency (the degree of influence the user has on the course of the narrative)." [21].

The usage of EPUB 3-based e-books in mobile environments [11] makes it possible to enhance these e-books with interactive features, including augmented reality and contextualization. Furthermore, connecting these e-books to the digital and physical world is key to making the storytelling more interactive.

Considering the above mentioned classification of IDN artifacts, a simple EPUB 3-based Hybrid Book modeling a fiction book with limited narrative agency (e.g., just flipping pages) can already be seen as an IDN artifact. Moreover, the Hybrid Book concept allows for more substantial interactivity features, and where these features can be realized/defined by the author or the designer of the story.

In the next section, we describe our prototype EPUB 3-based Hybrid Book.

\section{The Hybrid Book Concept}

Our research focuses on a Hybrid Book that takes the form of an e-book that facilitates interaction between the physical and the digital world. In particular, we aim to provide a generic data model to enable this interaction. In this section, we describe a prototype Hybrid Book that uses our generic data model to have interaction with devices in a smart living environment ${ }^{7}$.

Facilitating real-world interaction requires seamless collaboration between different e-book elements (e.g., story entities and emotions) and devices in smart living environments (e.g., sounds and lights). We believe that the proposed approach helps publishers to create an enjoyable reading experience. To create a prototype Hybrid Book, we selected a children's book, namely "Red Riding Hood" from Project Gutenberg ${ }^{8}$. Project Gutenberg is an initiative that consists of freely available online e-books in different formats such as PDF and EPUB. Next, we enriched the book, taking on the role of a designer of interactive story books. We aimed at influencing the digital and physical reading environment by manifesting different story entities and emotions via different human senses.

Fig. 1 demonstrates a high-level overview of the Hybrid Book setup. As can be seen in Fig. 1, the author or editor annotates the story entities and emotions that are relevant to the desired interactions, before distributing the book as an EPUB 3 file. When the book is read, the annotated data are extracted from the book and are broadcasted (e.g., using socket.io ${ }^{9}$ ) to all the smart device services via an e-reader (that is, an application for reading e-books). The smart devices need to be installed in the network used by the Hybrid Book. If any of the smart devices are missing, then an alert can for instance be shown in the pages of the

\footnotetext{
${ }^{7}$ We will make our EPUB 3-based prototype Hybrid Book available for download upon acceptance of this paper.

${ }^{8}$ http://www.gutenberg.org

${ }^{9}$ http://socket.io/
} 


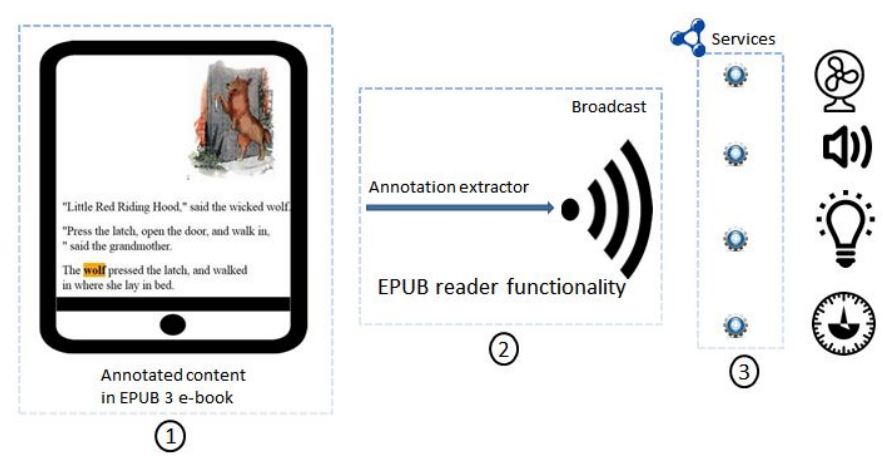

Fig. 1. High-level overview of the Hybrid Book setup: (1) story entities and emotions are annotated in the EPUB 3-based e-book; (2) the e-book reader extracts these annotations from the e-book and subsequently broadcasts these annotations; and (3) smart device services retrieve the broadcasted annotations, subsequently querying and reasoning about configuration data (i.e., data needed to activate the smart devices).

e-book. In addition, a specific service needs to be developed for each device. These services constitute a bridge between the Hybrid Book and the device. Furthermore, the data are interpreted by each device service, to determine the actions that need to be executed.

\subsection{Representation of Story Entities and Emotions}

We consider the author of a story as the designer of the Hybrid Book. Indeed, the author is the person who decides what actions need to be taken. In that regard, the desired interactions that an author wants to invoke must be inserted in the e-book at the correct position. To that end, we use HTML annotations using RDFa. These annotations define which changes are intended, and possibly, which action(s) should be performed. Fig. 4.1 shows a possible annotation for an entity. It shows how the word "wolf" is annotated with a "property" attribute, containing two customized items: "animal:wolf" and "emotion:afraid".

<span property="animal:wolf; emotion:afraid" > wolf $</$ span $>$

Fig. 2. The story entity "wolf" is annotated with a "property" attribute, containing two customized items: "animal:wolf" and "emotion:afraid".

\subsection{Triggering Actions Using Story Entities and Emotions}

The way we define different attributes is described below. These attributes are extendable if needed. Table 1 shows an example of matching entities to possible smart devices. 


\begin{tabular}{|l|c|c|c|}
\hline examples of story entities and emotions [32,26] & \multicolumn{2}{|c|}{ possible smart devices } \\
\hline & sound player & lights & thermostat \\
\hline emotion (e.g., afraid) & & $\checkmark$ & \\
\hline animal (e.g., wolf) & $\checkmark$ & $\checkmark$ & \\
\hline event (e.g., earthquake) & $\checkmark$ & & \\
\hline temperature (e.g., warm/cold) & & & $\checkmark$ \\
\hline
\end{tabular}

Table 1. Mapping story entities and emotions to possible devices.

For this research, we work with the six basic emotion terms proposed by Paul Ekman [9] : "Angry", "Afraid", "Disgusted", "Sad", "Surprised", and "Happy". We can associate specific colors with a "mood" or an emotion [25]: e.g., "Sad" can be associated with the color "blue". By expanding the story entities (e.g., animal) to their sub-entities (e.g., bird and wolf), we create a so-called Hybrid Book dataset that takes on the form of an an ontology ${ }^{10}$. This dataset consists of story entities, their corresponding data, and possible actions (e.g., "hasSound" as an action for a sound player and "hasColor" as an action for smart lights).

The e-reader extracts the annotations from any visible content (based on the size of the screen of the mobile devices used for reading). These extracted annotations (e.g., "animal:wolf") are then sent to services (e.g., over the Internet or a local network). Indeed, we assume that each smart device has a service that listens for incoming data. These services need to be implemented specifically for each device. It is necessary to have such services, because each device has its own functionality, and that functionality needs to be adapted to the proposed solution. For example, the Philips hue lights have the functionality of changing the color of lights. The proposed Hybrid Book needs to trigger the lights based on the annotated content. Thus, a service needs to bridge the gap between the Hybrid Book and the underlying APIs of smart devices. These services interpret the received data to derive desired actions, by querying them against our dataset. For example, when a story entity such as "wolf" has been annotated in the text, the process executed is as follows:

1. The e-reader extracts the annotated entity "wolf" from the book.

2. The e-reader converts the data related to the annotated story entity in RDF format as ":Wolf :Emotion :Afraid". The data are subsequently broadcasted to all connected devices.

3. All connected devices receive the broadcasted data.

4. The services decide what actions to take, based on a set of rules (e.g., (:Afraid rdf:type :Emotion, :hasColor :Orange), or (:Wolf rdf:type :Animal, :hasSound $:$ Wolf $))$ stored in the dataset. In fact, the services query the received data against our dataset using the Linked Data Fragments [37] server and client software. To that end, the $\mathrm{LDF}^{11}$ Client and Server are used for running SPARQL queries over Turtle data in our dataset. The resulting configuration

${ }^{10}$ We will make this ontology available online upon acceptance of this paper.

${ }^{11}$ https://github.com/LinkedDataFragments 
data (data that are required to activate the smart devices) will for instance be formatted as ":Action :hasSound :Wolf" for the audio player and ":Action :hasColor :Orange" for the smart lights. Furthermore, to get the desired result (e.g., a relevant sound) from online resources such as Spotify ${ }^{12}$, we need to have accurate search keywords. Based on the configuration data, the service of the sound player searches for wolf in Spotify and fetches a wolf sound.

5. Finally, the light service sends the action to the hue lights to change the color of the lights, and at the same time, the sound service sends the action to the audio player to play the corresponding sound.

\subsection{Authoring Hybrid Books}

In this context, we would like to make note that the creation of EPUB 3-based Hybrid Books should be cost-effective, thus requiring a user-friendly authoring environment. Ideally, creating a Hybrid Book is facilitated by an authoring environment that makes it possible for authors and publishers to indicate which elements of a story need to be annotated. Such an authoring environment is for instance discussed in [8], facilitating collaborative creation of enriched ebooks using EPUB 3, also enabling authors and publishers to design and create EPUB 3-based story books with annotations.

\section{Evaluation}

In this section, we describe the evaluation of our prototype EPUB 3-based Hybrid Book as an IDN artifact. Koenitz [18] discusses five aspects of IDN artifacts that are important: "narrative analysis", "interoperability between different implementations", "sustainability of digital artifacts", "author-centered view", and "user-focused perspective". The author argues that these key aspects need to be addressed in ongoing and future work in order to have successful methods and artifacts in the field. In what follows, we evaluate our Hybrid Book concept, focusing on the aforementioned key aspects of IDN artifacts, with the exception of "narrative analysis", given that this aspect is not within our domain of expertise.

- Interoperability between different implementations: This aspect refers to the use of technical standards for, e.g., data exchange between different implementation platforms. As described in the Hybrid Book section, we use Turtle as our standard format for data exchange. In addition, the EPUB 3 format itself is an open standard for digital publications. Ghaem Sigarchian et al. [11] concluded that this open publication standard allows for interoperability between different mobile devices. In what follows, we list a number of relevant EPUB 3 capabilities: "sensor data utilization in mobile devices", "data transfer to servers", "connecting to local and remote networks", and "discoverable content".

\footnotetext{
12 https://www . spotify.com/
} 
- Sustainability of digital artifacts: This is a key design aspect that can be achieved by following standards, making the artifacts sustainable. All technologies used in the implementation of our Hybrid Book are based on open standards, thus preventing "reinventing the wheel" in future research.

- Author-centered view: This key aspect refers to involving (multiple) authors of traditional narratives in IDNs, making it possible for these authors to easily design their own interactive digital story. They can thus apply their own preferences in terms of actions and emotions (that is, they can transfer their own vision to the audience).

- User-focused perspective: Given that this aspect is key to creating exciting narrative experiences, we organized a usability test in a lab environment at a research center, to quantify the level of reader enjoyment. This seemed the most appropriate method to have a profound evaluation of the concept from both parents and children. A usability test is intended to determine the

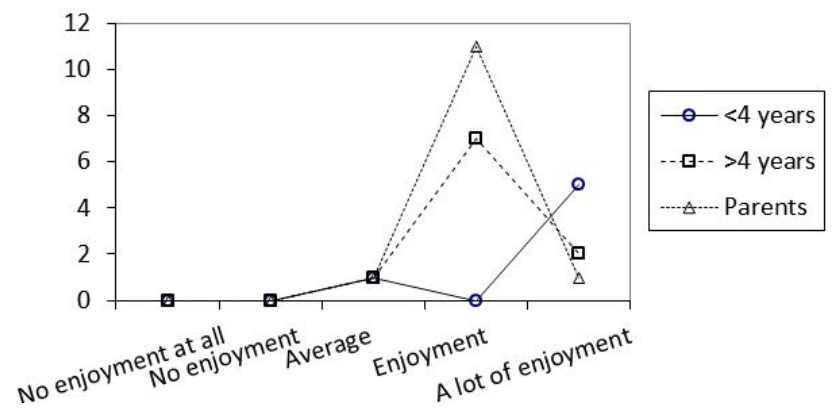

Fig. 3. Likert scale on the enjoyment of the book by children younger than 4 years, older than 4 years and parents $(n=$ number of people).

extent an interface facilitates the ability of a user to complete routine tasks. Typically the test is conducted with a group of potential users either in a usability lab, remotely (using e-meeting software and telephone connection), or on-site with portable equipment. Users are asked to complete a series of routine tasks. Sessions are recorded and analyzed to identify potential areas for improvement to innovation. This approach is based on usability evaluation methods $[33,12,22,1]$. When focusing on the testing of the usability of an innovation, it is recommended to do the test in a lab environment $[38,16]$. The test took place in June 2015. The purpose of the test was to have a user validation of the Hybrid Book concept and how a Hybrid Book can be connected to smart devices in a room while reading a story. In total, 29 participants (13 parents and 16 children) took the test and more tests will be conducted after the submission deadline of this paper. Every session took about half an hour. The story consisted of five pages so it did not take too long to read the story. Afterward, the children were asked to color a smiley 
matching their enjoyment and they were given an incentive. At the same time, the parents were interviewed.

The children were all very enthusiastic about the hybrid book, while parents were more critical. The scale we used to capture the opinion of the children is based on the Smileyometer [29], a discrete Likert Scale. In general, the children liked reading the prototype Hybrid Book. The youngest children liked this type of book the most. Parents were also very enthusiastic about the enjoyment experience of the concept. They also saw educational opportunities for the book. The majority of the parents $(n=13)$ were willing to pay between 5 and 15 euros for a Hybrid Book. Only one parent would pay 1 euro for a story. All parents said they would use the technology. We used a single intention question in order to map the adoption intention of the parents. We do have to be critical with this approach because it tends to stimulate overestimation $[7,6]$. All of the parents answered that at least the chance is high they would use this type of book.

The most important issue is a lack of willingness to adopt smart technologies. Because the technology is still in a concept phase, it was difficult for every parent to imagine all the future possibilities for interactions with smart technologies. This usability test gave us a first insight in the user evaluation of the concept. It is advisable to further test and adapt the concept with future users to get more in-depth insights.

\section{Conclusions and Future Work}

In this paper, we discussed the creation of interactive story books, using Digital Publishing and Semantic Web technologies. In particular, we investigated how semantic annotations can be used to author interactive story books, i.e. so-called Hybrid Books. Furthermore, we evaluated a prototype Hybrid Book by considering key aspects of IDN artifacts. We found that our Hybrid Book contributes to an enjoyable reading experience. In addition, our Hybrid Book is an interoperable and sustainable IDN artifact, making it possible for authors to apply their own preferences by inserting semantic annotations into Hybrid Books.

We can identify a number of directions for future research. First, to alleviate the burden of manual annotation, we could explore the use of an automatic story entity and emotion recognition web service for already existing story books. Second, the current version of our Hybrid Book can be improved with more interactivity features, including read aloud and eye tracking functionality.

\section{References}

1. Albert, W., Tullis, T.: Measuring the User Experience: Collecting, Analyzing, and Presenting Usability Metrics. Newnes (2013)

2. Anastasiades, S.P.: The Future of the Book, the Book of the Future. In: Advanced Learning Technologies, 2003. Proceedings. The 3rd IEEE International Conference on. pp. 246-247. IEEE (2003) 
3. Baggia, Paolo and Pelachaud, Catherine and Peter, Christian and Zovato, Enrico: Emotion Markup Language (EmotionML) 1.0 W3C Recommendation 22 May 2014 Accessed June 15th, 2015. http://www.w3.org/TR/emotionml/

4. Bartneck, C., Hu, J., Salem, B., Cristescu, R., Rauterberg, M.: Applying Virtual and Augmented Reality in Cultural Computing. IJVR 7(2), 11-18 (2008)

5. Beckett, David and T. Berners-Lee, Tim and E. Prud'hommeaux, Eric and Carothers, Gavin: RDF 1.1 Turtle Terse RDF Triple Language W3C Recommendation 25 February 2014. Accessed June 15th, 2015. http://www.w3.org/TR/turtle/

6. De Marez, L.: Diffusie van ICT-Innovaties: Accurater Gebruikersinzicht voor Betere Introductiestrategieën. Ph.D. thesis, Ghent University (2006)

7. De Marez, L., Verleye, G.: Innovation Diffusion: The need for more Accurate Consumer Insight. Illustration of the PSAP Scale as a Segmentation Instrument. Journal of Targeting, Measurement and Analysis for Marketing 13(1), 32-49 (2004)

8. De Meester, B., De Nies, T., Ghaem Sigarchian, H., Vander Sande, M., Van Campen, J., Van Impe, B., De Neve, W., Mannens, E., Van de Walle, R.: A Digital-First Authoring Environment for Enriched e-Books using EPUB 3. Information services \& use 34(3-4), 259-268 (2014)

9. Eckman, P.: Universal and Cultural Differences In facial Expression of Emotion. In: Nebraska symposium on motivation. vol. 19, pp. 207-284. University of Nebraska Press Lincoln (1972)

10. Ghaem Sigarchian, H., De Meester, B., De Nies, T., Verborgh, R., De Neve, W., Mannens, E., Van de Walle, R.: EPUB3 for Integrated and Customizable Representation of a Scientific Publication and its Associated Resources. In: Proceedings of the 4th Workshop on Linked Science (2014)

11. Ghaem Sigarchian, H., De Meester, B., De Nies, T., Verborgh, R., Salliau, F., De Neve, W., Mannens, E., Van de Walle, R.: Towards Making EPUB 3-based e-TextBooks a First-Class Mobile Learning Environment. (2015)

12. Hassenzahl, M., Tractinsky, N.: User experience-a Research Agenda. Behaviour \& information technology 25(2), 91-97 (2006)

13. Herman, Ivan and Adida, Ben and Sporny, Manu and Birbeck, Mark: RDFa 1.1 Primer - Third Edition Rich Structured Data Markup for Web Documents W3C Working Group Note 17 March 2015. Accessed June 15th, 2015. http://www.w3. org/TR/rdfa-primer/

14. Hickson, Ian: HTML Microdata. Technical Report, W3C, October 2013. Accessed June 15th, 2015. Accessed June 15th, 2015. http://www.w3.org/TR/microdata/

15. IDPF: Electronic Publication, Version 3. http://idpf .org/epub/30

16. Kjeldskov, J., Skov, M.B., Als, B.S., Høegh, R.T.: Is It Worth the Hassle? Exploring the Added Value of Evaluating the Usability of Context-Aware Mobile Systems in the Field. In: Mobile Human-Computer Interaction-MobileHCI 2004, pp. 61-73. Springer (2004)

17. Klyne, Graham and J. Carroll, Jeremy and McBride,Brian: RDF 1.1 Concepts and Abstract Syntax. Technical Report, W3C, February 2014. Accessed June 15th, 2015. http://www.w3.org/TR/rdf11-new/

18. Koenitz, H.: Five Theses for Interactive Digital Narrative. In: Interactive Storytelling, pp. 134-139. Springer (2014)

19. Koenitz, H.: Towards a Specific Theory of Interactive Digital Narrative. Interactive Digital Narrative: History, Theory and Practice p. 91 (2015)

20. Koenitz, H., Chen, K.J.: Genres, Structures and Strategies in Interactive Digital Narratives-Analyzing a Body of Works Created in ASAPS. In: Interactive Storytelling, pp. 84-95. Springer (2012) 
21. Koenitz, H., Haahr, M., Ferri, G., Sezen, T.I., Sezen, D.: Mapping the Evolving Space of Interactive Digital Narrative-From Artifacts to Categorizations. In: Interactive Storytelling, pp. 55-60. Springer (2013)

22. Law, E., Scapin, D., Cockton, G., Stary, M., Winckler, M.: Maturation of Usability Evaluation Methods: Retrospect and Prospect. In: COST294-MAUSE Closing Conference Proceedings(2009) (2009)

23. Liljedahl, M.: Sound for Fantasy and Freedom. Game Sound Technology and Player Interaction: Concepts and Developments pp. 22-44 (2011)

24. Nakevska, M., Funk, M., Hu, J., Eggen, B., Rauterberg, M.: Interactive Storytelling in a Mixed Reality Environment: How Does Sound Design and Users Preknowledge of the Background Story Influence the User Experience? In: Interactive Storytelling, pp. 188-195. Springer (2014)

25. Nijdam, A.N.: Mapping Emotion to Color. )^(Eds.):Book Mapping emotion to color(2009, edn.) pp. 2-9 (2009)

26. Osondu, C., Wei, W.: Named Entity Classification

27. PrudHommeaux, Eric and Seaborne, Andy: SPARQL Query Language for RDF. W3C Recommendation 15 (2008). Accessed June 15th, 2015. http://www.w3.org/ TR/rdf-sparql-query/

28. R. Gruber, T.: A Translation Approach to Portable Ontology Specifications. Knowledge acquisition 5(2), 199-220 (1993)

29. Read, J.C., MacFarlane, S.: Using the Fun Toolkit and Other Survey Methods to Gather Opinions in Child Computer Interaction. In: Proceedings of the 2006 conference on Interaction design and children. pp. 81-88. ACM (2006)

30. Sánchez-Rada, J.F., Iglesias, C.A.: Onyx: A Linked Data Approach to Emotion Representation. Information Processing \& Management (2015)

31. Seadle, M., Vassiliou, M., Rowley, J.: Progressing the Definition of e-book. Library Hi Tech 26(3), 355-368 (2008)

32. Sekine, S., Sudo, K., Nobata, C.: Extended Named Entity Hierarchy. In: LREC (2002)

33. Shaw, C., Ivens, J.: Building Great Customer Experiences. Palgrave Macmillan (2002)

34. Smith, Michael K. and Welty, Chris and L. McGuinness, Deborah: OWL Web Ontology Language Guide: W3c Recommendation 10 February 2004, 2004. Accessed June 15th, 2015. http://www.w3.org/TR/2004/REC-owl-guide-20040210/

35. Thoma, G.R., Ford, G., Chung, M., Vasudevan, K., Antani, S.: Interactive Publications: Creation and Usage. In: Electronic Imaging 2006. pp. 607603-607603. International Society for Optics and Photonics (2006)

36. Verborgh, R., Hartig, O., De Meester, B., Haesendonck, G., De Vocht, L., Vander Sande, M., Cyganiak, R., Colpaert, P., Mannens, E., Van de Walle, R.: Querying Datasets on the Web with High Availability. In: The Semantic Web-ISWC 2014, pp. 180-196. Springer (2014)

37. Verborgh, R., Vander Sande, M., Colpaert, P., Coppens, S., Mannens, E., Van de Walle, R.: Web-Scale Querying trough Linked Data Fragments. In: Proceedings of the 7th Workshop on Linked Data on the Web (2014)

38. Vermeeren, A.P., Law, E.L.C., Roto, V., Obrist, M., Hoonhout, J., VäänänenVainio-Mattila, K.: User Experience Evaluation Methods: Current State and Development Needs. In: Proceedings of the 6th Nordic Conference on Human-Computer Interaction: Extending Boundaries. pp. 521-530. ACM (2010) 